\title{
AGAINST GRAMMATICAL COMPUTATION OF SCALAR IMPLICATURES
}

Benjamin Russell (brussell@brown.edu)

Brown University, Department of Cognitive and Linguistic Sciences

presented at the workshop on Implicature and Conversational

Meaning, 16-20 August 2004, Nancy.

\section{Introduction}

Scalar implicatures (SIs), according to a theoretical tradition that goes back to Grice (1975) and is elaborated and formalized by Horn (1972, 1989), Gazdar (1979), Sauerland (2004), and Blutner (2004), have been understood as inferences of the following (somewhat abbreviated) form:

(1) Speaker A said $\phi$, and could as easily have said $\psi$, which is stronger. Because $\mathrm{A}$ is cooperative, she makes the strongest true statement possible, so $\psi$ can't be true.

Recently, Chierchia (to appear) (henceforth C) has proposed a radical departure from the Gricean theory: SIs, he argues, are not the product of rational behavior between cooperative conversants as in (1), but rather are computed automatically in the grammar by means of special semantic composition rules and lexical scales. He argues that reformulating the process of SI computation in this way has two advantages: it accounts for the generation of a class of SIs that the Gricean theory doesn't; and negative polarity item licensing, a grammatical process, is sensitive to SIs, which are available to grammatical processes under his theory but not under a Gricean theory. I will argue that, despite the ingenuity of C's formalism, neither of his arguments for adopting it is valid. I show that a Gricean theory can account for each of the implicatures $\mathrm{C}$ identifies, and that negative polarity item licensing is not affected by SIs. Thus, the empirical predictions of C's theory, which requires the stipulation of a secondary semantic composition system, do not surpass the empirical predictions of the Gricean theory, which assumes only that speakers are cooperative.

\section{Gricean computation of implicatures of complex sentences}

C's first argument consists in identifying a range of observed SIs that are not obviously generated by Gricean principles. The most compelling of these are apparently embedded implicatures of the following form.

(2) George believes some of his advisors are waiting.

a. $\rightsquigarrow$ George believes not all of his advisors are waiting.

b. $\quad \Varangle \rightarrow$ It is not the case that George believes all of his advisors are waiting. 
$\mathrm{C}$ argues that Gricean reasoning cannot generate the observed implicature in (2a); if SIs are derived by the kind of reasoning in (1), hearers can only make conclusions about the negation of competing utterances, not embedded clauses within those utterances, and so they should only infer $(2 \mathrm{~b})$. On the other hand, in C's system, the implicature of some of his advisors are waiting is computed to give a strong meaning of some of his advisors are waiting and not all of his advisors are waiting, which subsequently combines with George believes to give a strong meaning of George believes some of his advisors are waiting and not all of his advisors are waiting. Thus, by grammaticizing the process of SI computation, $\mathrm{C}$ is able to correctly compute embedded SIs like (2a).

But notice that $(2 \mathrm{a})$ follows from $(2 \mathrm{~b})$ in every context where George has SOME belief about whether all of his advisors are waiting: if it is not the case that George believes that all of his advisors are waiting, but George has some belief about whether all of his advisors are waiting, then George must believe that not all of his advisors are waiting. Furthermore, in a context where this assumption is explicitly denied, the embedded implicature is not generated. For example, if George has four advisors and has an appointment with Dick and Donald, but has no idea whether his other two advisors, Colin and Condoleeza, will be waiting for him, the utterance George believes some of his advisors are waiting for him only implies (2b), not (2a). The enrichment of weak implicatures with contextual inferences in a global, Gricean framework correctly mirrors the observed fine-grained context-sensitivity of scalar implicatures. And the fact that Gricean inferencing CAN generate ostensibly embedded implicatures as well as weaker global implicatures obviates the need $\mathrm{C}$ tries to establish for a compositional system of implicature computation.

C further argues that the Gricean theory incorrectly predicts (3) implicates $(3 b)$, not $(3 a)$.

(3) George ate some of the fries or the apple pie.

a. $\rightsquigarrow$ It is not the case that George ate all of the fries.

b. $\quad \varkappa_{\rightarrow}$ It is not the case that George ate all of the fries or the apple pie.

Following Sauerland (2004), I propose that a reconsideration of the epistemic status of implicatures provides a solution to this problem; I reiterate Sauerland's insights about sentences like (3) here in general Gricean terms. Sauerland follows Horn (1989) and Soames (1982) in recognizing that the Gricean reasoning for SI computation in (1) is more accurately represented as (4).

(4) Speaker A said $\phi$, and could as easily have said $\psi$, which is stronger. Because A is cooperative, she makes the strongest true statement possible, so A must not know $\psi$ is true $(\neg \mathbf{K} \psi)$. 
Under this view, scalar implicatures are weaker inferences of the form $\phi \rightsquigarrow$ $\neg \mathbf{K} \psi$, rather than $\phi \rightsquigarrow \mathbf{K} \neg \psi$. Implicatures are only strengthened to $\mathbf{K} \neg \psi$ when the hearer assumes that the speaker knows WHETHER $\psi$ is true $(\mathbf{K} \psi \vee \mathbf{K} \neg \psi)$. This increased fidelity to Grice's theory provides a solution to C's puzzle, for (3) now has the implicature in (5).

(5) $\neg \mathbf{K}$ George ate all of the fries or the apple pie.

And (5) entails the following two epistemic facts about the speaker.

$$
\begin{aligned}
& \text { a. } \quad \neg \mathbf{K} \text { George ate all of the fries. } \\
& \text { b. } \quad \neg \mathbf{K} \text { George ate the apple pie. }
\end{aligned}
$$

In contexts where the hearer can assume the speaker knows whether George ate all the fries, (6a) can be strengthened, yielding the desired implicature in (3a). In all other contexts, (6a) is the strongest implicature drawn, which is very much in keeping with my intuitions.

This analysis presents two potentially contentious points. First, do hearers actually freely strengthen entailments of implicatures? Sauerland's specific formalism prevents this question from arising - but in the general Gricean framework employed here, I propose that if $\neg \mathbf{K}$ implicatures can be strengthened, their entailments should also be strengthenable. The logic of this is as follows: the process of inferring an implicature is no more and no less than attributing a mental state to your conversational partner. So, if $\mathrm{B}$ infers that $\mathrm{A}$ has the state $\neg \mathbf{K} \psi$, and $\phi \subset \psi$, then A must also have the state $\neg \mathbf{K} \phi$ (otherwise, A would have to know $\psi$ ). Then, B can infer $\mathbf{K} \neg \phi$ whenever B has reason to think that $\mathrm{A}$ is well-informed about $\phi$. The second contentious question is: why isn't (6b) strengthened to the obviously undesirable inference (7)?

$$
\mathbf{K} \neg \text { George ate the apple pie. }
$$

Sauerland finds the answer to this in another set of implicatures of (3): the implicatures Gazdar referred to as clausal implicatures, which, in the case of disjunction, are of the form $p \vee q \rightsquigarrow \neg \mathbf{K} p \wedge \neg \mathbf{K} q$. Following Sauerland, I think clausal implicatures may generally be understood as a class of scalar implicatures: both $p$ and $q$ are stronger than (entail) $p \vee q$, and $p$ and $q$ are each certainly easier to say than $p$ or $q$, so if a speaker has chosen to use $p$ or $q$, she must not know that either $p$ or $q$ is true. Thus, the weak implicatures in (8) should be generated with the weak implicatures in (5).

a. $\neg \mathbf{K}$ George ate some of the fries.

b. $\neg \mathbf{K}$ George ate the apple pie.

This set of implicatures is enough to "block" the strengthening of (6b) to (7). The derivation is as follows:
a. $\quad \mathbf{K}$ (George ate some of the fries $\vee$ George ate the apple pie) (from (3))
b. $\quad \mathbf{K}(\neg$ George ate the apple pie $\rightarrow$ George ate some of the fries) 
(equivalent to $(9 \mathrm{a})$ )

c. $\quad \mathbf{K} \neg$ George ate the apple pie $\rightarrow \mathbf{K}$ George ate some of the fries (from (9b) by axioms of epistemic logic)

d. $\neg \mathbf{K}$ George ate some of the fries $\rightarrow \neg \mathbf{K} \neg$ George ate the apple pie (contrapositive of $(9 \mathrm{c})$ )

e. $\quad \neg \mathbf{K}$ George ate some of the fries (from (8a))

f. $\quad \neg \mathbf{K} \neg$ George ate the apple pie (modus ponens)

So (7) is blocked and the observed implicature (3a) is generated by the Gricean theory without any undesirable consequences. The Gricean theory is again capable of generating the right implicatures for complex sentences, and C's argument that the observed implicatures of sentences like (2) and (3) are not generated by a global theory is thus countered.

\section{Scalar Implicatures and Intervention}

C's second argument for a local theory is that an adequate treatment of intervention in negative polarity item (NPI) licensing depends on SIs. NPIs (like any) are licensed by downward-entailing operators - an operator $f$ is downward entailing if for any $\alpha \subset \beta, f(\alpha) \supset f(\beta)$; examples are not, doubt, and if (Ladusaw 1979). Intervention is an exception to the licensing of NPIs by downward-entailing operators: any is not licensed by downward-entailing operators if it appears in the scope of every or as one of the conjuncts of and:

a. *Condoleeza didn't drink any Coke and a milkshake. (cf. Condoleeza didn't drink any Coke or a milkshake.)

b. *Dick doubts everyone ate any McNuggets. (cf. Dick doubts anyone ate any McNuggets.)

Though semantic theories of NPI licensing have been very successful, C is among the first to propose a semantic solution to the intervention puzzle (but cf. Honcoop (1996)). C's solution is predicated on Kadmon and Landman's (1993) semantics of any, whereby any means approximately the

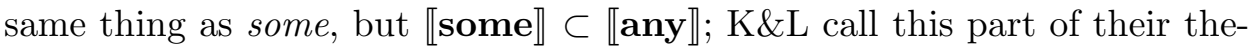
ory Widening. The second part of K\&L's theory is a condition they call Strengthening: given a sentence $S_{a n y}$ containing any, it must be the case that $S_{a n y} \subset S_{\text {some }}$, where $S_{\text {some }}$ is identical to $S_{\text {any }}$, except some is substituted for any. Widening and Strengthening combined predict that any will be licensed by downward entailing operators. Crucially, this licensing must be grammatical, because Widening and Strengthening make the wrong predictions about any-licensing at the global level. In particular, sentences like (11) do not globally satisfy Strengthening.

(11) If Colin doesn't have any money, he can’t buy a Big Mac. 
Because both if and not are downward-entailing, any appears globally in an upward-entailing environment in (11). So any must be licensed before it the expression containing it combines with if, while it still satisfies K\&L's Strengthening condition. This conclusion that NPIs must be grammatically licensed is the foundation of Chierchia's argument that his SI-based solution to the intervention puzzle suggests SIs are grammatically computed.

C's NPI theory assumes K\&L are basically correct but modifies their Strengthening condition, incorporating implicature-enriched strong meanings. A strong meaning is the conjunction of the regular semantics of an expression with its locally-computed scalar implicatures; given an expression $\alpha$, the strong meaning of $\alpha$ is notated $\alpha^{s}$. C's Strong Strengthening condition is $S_{\text {any }} \subset S_{\text {some }}^{s}$; in prose, the condition is that the meaning of a sentence with any must be stronger than (entail) the strong meaning of the corresponding sentence with some. Now, in (10a), $S_{\text {some }}^{s}=$ Condoleeza didn't drink some Coke and a milkshake, but she drank some coke or a milkshake, so, in particular, for any to be licensed, $S_{a n y}$-i.e. (10a) - must entail Condoleeza drank some Coke or a milkshake. $S_{a n y}$ does not entail this, so Strong Strengthening is not satisfied, and (10a) is ungrammatical.

Notice that Strong Strengthening requires $S_{a n y}$ to entail not only $S_{\text {some }}$, but also the implicatures of $S_{\text {some }}$. And, as seen in (10a), both $S_{a n y}$ and

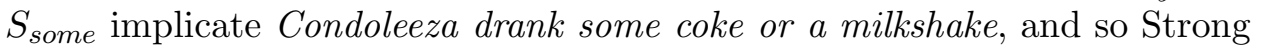
Strengthening here is actually a requirement that the sentence with any entail its own SI. In fact, this is the case for all the intervention cases C discusses, and can be proved to be the case for a broad class of intervention cases. And sentences do not entail their own scalar implicatures, by definition. So Strong Strengthening amounts to a restriction that any may not be licensed in sentences with scalar implicatures. That is, in C's theory, and and every are interveners simply because they have SIs when embedded below downward-entailing operators.

This leads to my final conclusion about intervention. C's theory predicts that every item that has SIs when embedded below a downward-entailing operator will be an intervener. So, in addition to and and every, the set of interveners should include every strong scalar term, like necessarily, excellent, and numerals (if you believe in the Gricean at least $n$ treatment of numerals) because no sentence with one of these items below a single downward-entailing operator will satisfy Strong Strengthening. But this prediction is contradicted by empirical data:

a. Donald won't necessarily eat any cheeseburgers.

b. George doubts anyone ate a Big Mac and a Quarter Pounder.

In (12a), $S_{\text {some }}$ implies Donald will possibly eat some cheeseburgers. So Strong Strengthening requires that $S_{a n y}$-i.e. (12a) - entail that Donald will possibly eat some cheeseburgers. But, of course, this is an implicature, and not an entailment. Likewise, in (12b), $S_{\text {some implies George doesn't }}$ 
doubt someone ate a Big Mac or a Quarter Pounder, which, in turn, is not entailed by $S_{a n y}$, and so Strong Strengthening is not satisfied. This example is especially striking because it shows that and is only an intervener when any appears in one of its conjuncts - not in the broader set of circumstances where the implicatures of and are computed before any is licensed by a downward-entailing operator, as $\mathrm{C}$ predicts. C's theory thus incorrectly predicts that both sentences in (12) are ungrammatical, suggesting that it is not the SIs associated with and and every that are responsible for their behavior as interveners.

\section{Conclusion}

C's proposal to grammaticize the computation of SIs is a radical departure from Gricean pragmatics. If $\mathrm{C}$ is right, SIs have nothing to do with rational principles of cooperation in conversation. Instead, this broad class of inferences that can be explained by the simple assumption that speakers cooperate is redundantly generated by a stipulated grammatical mechanism. C's argument for the radical shift to a grammatical theory of implicature computation depends on two claims. First, he claims that observed implicatures of embedded sentences are predicted by his local theory but not by global theories. I've argued that a very general global Gricean theory accounts for all such implicatures. Second, he develops a theory of intervention in NPI licensing that depends on grammatically computed SIs; I've argued that any theory that depends on SIs to exclude intervention cases is too stringent, failing to license any in many grammatical sentences. I conclude that the departure from Gricean pragmatics that Chierchia proposes is unmotivated.

\section{References}

Blutner, R.: 2004, Pragmatics and the lexicon, in L. Horn and G. Ward (eds), Handbook of Pragmatics, Blackwell.

Chierchia, G.: to appear, Scalar implicatures, polarity phenomena, and the syntax/pragmatics interface, in A. Belletti (ed.), Structures and Beyond, Oxford University Press.

Gazdar, G.: 1979, Pragmatics: Implicature, Presupposition, and Logical Form, Academic Press, New York.

Grice, H. P.: 1975, Logic and conversation, in D. Davidson and G. Harman (eds), The Logic of Grammar, Dickenson Publishing Co., Encino, California, pp. 64-75.

Honcoop, M.: 1996, Dynamic excursions on weak islands, PhD thesis, HIL Universiteit Leiden.

Horn, L. R.: 1972, On the Semantic Properties of the Logical Operators in English, Ph.D. dissertation, University of California at Los Angeles, Los Angeles, California.

Horn, L. R.: 1989, A Natural History of Negation, Chicago University Press, Chicago.

Kadmon, N. and Landman, F.: 1993, Any, Linguistics and Philosophy 16(4), 353-422. 
Ladusaw, W. A.: 1979, Polarity Sensitivity as Inherent Scope Relations, PhD thesis, University of Texas, Austin.

Sauerland, U.: 2004, Scalar implicatures in complex sentences, Linguistics and Philosophy 27, 367-391.

Soames, S.: 1982, How presuppositions are inherited: A solution to the projection problem, Linguistic Inquiry 13, 483-545. 